\title{
Revisões sistemáticas da literatura: Uma súmula para as ciências sociais
}

\section{Systematic literature reviews: A summary for the social sciences}

\author{
Nino Fonseca \\ Instituto Politécnico de Viana do Castelo, Portugal \\ ninomf@estg.ipvc.pt
}

Marcelino Sánchez-Rivero

Universidade da Extremadura, Espanha

sanriver@unex.es

\section{Resumo}

\begin{abstract}
Este trabalho tem como objetivo fazer uma apresentação sucinta, embora completa e rigorosa, das várias etapas de uma revisão sistemática da literatura, tendo em vista a sua aplicação no contexto das ciências sociais. Para o efeito, propomos a integração do enquadramento desenvolvido por Cooper (1982, 2010) e Cooper e Hedges (2009) e do conjunto de contributos sugeridos por vários outros autores recentes. As orientações apresentadas são suscetíveis de aplicação imediata por parte dos investigadores interessados.
\end{abstract}

Palavras-chave: revisões sistemáticas; meta-análise; ciências sociais.

\begin{abstract}
This article aims to make a succinct, but thorough and rigorous presentation of the various stages of a systematic review of the literature, with a view to its application in the context of the social sciences. To this end, we propose an integration between the framework developed by Cooper (1982, 2010) and Cooper and Hedges (2009) and a set of contributions suggested by several other recent authors. The guidelines presented are open to immediate application by interested researchers.
\end{abstract}

Keywords: systematic reviews; meta-analysis; social sciences.

\section{Introdução}

Neste trabalho debruçamo-nos sobre as metodologias de revisão sistemática da literatura, com uma ênfase especial sobre a sua aplicação no contexto das ciências sociais. A principal limitação e, ao mesmo tempo, crítica às revisões narrativas tradicionais diz respeito à forte presença de um viés de subjetividade, na medida em que, por um lado, o leque de estudos incluídos e excluídos depende das preferências do autor da revisão, que consegue encontrar, quase sempre, uma justificação razoavelmente "consensual” para as suas opções. Por outro lado, na presença de uma literatura com resultados fortemente heterogéneos pode ser 
muito difícil conciliar as respetivas conclusões de uma forma suficientemente objetiva e universal. Dito de outra forma, há sempre margem para interpretações e reconciliações alternativas dos resultados da literatura. As revisões sistemáticas da literatura permitem mitigar ou, até mesmo, ultrapassar estas dificuldades. Como tal, neste trabalho o nosso objetivo é descrever de uma forma sucinta, embora completa e rigorosa, as várias etapas subjacentes à correta aplicação deste expediente. Por esta via, pretendemos motivar os investigadores das ciências sociais a recorrer de forma mais intensiva às revisões sistemáticas da literatura, que já gozam de um forte prestígio noutras áreas do conhecimento, em particular nas ciências médicas, naturais e da saúde (Higgins \& Green, 2008; Moher et al., 2009).

A pesquisa de artigos e outras publicações académicas na base de dados Scopus com a frase "systematic review" no título, no resumo ou nas palavras-chave devolveu os resultados apresentados no Quadro 1. O crescimento exponencial da aplicação da metodologia de revisão sistemática da literatura é bastante evidente, sobretudo na área dos Negócios, Gestão e Contabilidade, com $37,6 \%$ de crescimento médio anual do número de trabalhos publicados entre 2000 e 2019.

Quadro 1. Evolução da aplicação de revisões sistemáticas da literatura

\begin{tabular}{llllll}
\hline \hline & $\mathbf{2 0 0 0}$ & $\mathbf{2 0 0 5}$ & $\mathbf{2 0 1 0}$ & $\mathbf{2 0 1 5}$ & $\mathbf{2 0 1 9}$ \\
\hline \hline Todas as áreas & 784 & 6309 & 7974 & 22918 & 26370 \\
Ciências Sociais & 25 & 131 & 216 & 801 & 1627 \\
Negócios, Gestão e Contabilidade & 1 & 18 & 30 & 161 & 428 \\
Economia, Econometria e Finanças & 1 & 2 & 17 & 75 & 140 \\
Viagens, Turismo e Hospitalidade & 3 & 10 & 20 & 68 & 110 \\
\hline \hline
\end{tabular}

A literatura está repleta de exemplos de aplicação desta metodologia. Dentro dos trabalhos mais citados e emblemáticos, encontramos estudos sobre inovação organizacional e networking (Crossan \& Apaydan, 2010; Pittaway, Robertson, Munir, Denyer \& Neely, 2004), educação para o empreendedorismo (Pittaway \& Cope, 2007), relações universidadeindústria (Perkman et al., 2013), responsabilidade social das empresas (Peloza \& Shang, 2011), microcrédito (van Rooyen, Stewart \& de Wet, 2012), eficiência dos mercados (Lim \& Brooks, 2011), gestão da cadeia de valor em turismo (Zhang, Song \& Huang, 2009), desporto e turismo (Weed, 2006), inovação em hospitalidade em turismo (Gomezelj, 2006), entre outros.

As revisões sistemáticas da literatura constituem uma etapa prévia e indispensável dos estudos de meta-análise, os quais, por sua vez, e em conjunto com aquelas revisões, permitem apresentar verdadeiros balanços do conhecimento disponível no âmbito das questões de investigação em causa. Neste trabalho a nossa atenção reside, pois, na descrição das caraterísticas das revisões sistemáticas da literatura tendo em vista a sua aplicação no âmbito das ciências sociais que façam uso de métodos de análise quantitativa.

$\mathrm{Na}$ secção seguinte fazemos uma comparação entre as abordagens das revisões narrativas e das revisões sistemáticas da literatura. Na secção 3 passamos à descrição e discussão das várias etapas das revisões sistemáticas. Para o efeito, complementamos o enquadramento geral avançado por Cooper (1982, 2010) e Cooper e Hedges (2009) com 
recomendações de outros autores tendo em vista a realização de revisões sistemáticas no contexto das ciências sociais. Na última secção tecemos algumas considerações finais.

\section{Revisões sistemáticas vs. revisões narrativas da literatura}

Nesta secção descrevemos os princípios gerais de revisão sistemática da literatura, tal como propostos por Cooper $(1982,2010)$. As revisões sistemáticas da literatura distinguem-se das revisões narrativas pelo facto de abordarem o processo de revisão de uma forma mais rigorosa, transparente e suscetível de replicação por outros autores. São tanto mais úteis quantas mais das seguintes circunstâncias se verificarem: o tema específico em análise é caraterizado pela existência de um elevado número de estudos (Hunter, Schmidt \& Hunter, 1979, por exemplo, passam em revista 866 análises empíricas, embora não existam limites, nem inferiores nem superiores, à quantidade de estudos que podem ser considerados numa mesma revisão sistemática); os resultados obtidos pelos vários estudos são inconsistentes (ou seja, as suas conclusões são contraditórias); é possível que as inconsistências observadas decorram da intervenção de fatores moderadores (como a dimensão ou natureza da amostra, as técnicas estatísticas utilizadas, entre muitos outros fatores possíveis).

De facto, as revisões narrativas, que primam por serem flexíveis do ponto de vista da respetiva abordagem metodológica são, por isso mesmo, fortemente subjetivas. Nesse sentido, não é incomum diferentes revisões de literatura sobre um determinado tópico chegarem a conclusões gerais divergentes (Suri, 2000; Barnes \& Bero, 1998). Isso pode acontecer porque os conjuntos de estudos incluídos nas revisões narrativas são diferentes, ou porque, mesmo na eventualidade de serem revistos os mesmos estudos, existem diferenças subjetivas no que diz respeito à integração dos respetivos resultados. Nas revisões narrativas, estes problemas tendem a agravar-se à medida que o número de estudos revistos aumenta.

Pelo contrário, as revisões sistemáticas obedecem a um procedimento razoavelmente padronizado (Cooper, 1982, 2010; Shadish, 2015), pelo que são menos permeáveis a considerações de caráter subjetivo. Ou seja, são mais objetivas, mais eficientes na análise simultânea de um grande número de estudos e, também, mais facilmente replicáveis. Não significa isto que estejam isentas de limitações. Por um lado, é sempre difícil capturar as diferenças qualitativas existentes entre os diferentes estudos empíricos. Trata-se de saber, por exemplo, se se deve dar igual importância aos estudos oriundos de revistas com diferentes significados em termos de fator de impacto ou de prestígio.

Por outro lado, alguns estudos empíricos são pouco claros na forma como apresentam os respetivos resultados e conclusões. É o que acontece, por exemplo, quando os investigadores concluem a favor da aceitação ou da rejeição de determinadas hipóteses estatísticas, mas não indicam, com precisão, quais foram as probabilidades de significância (i.e. valores-p) que ditaram essas conclusões.

Adicionalmente, por vezes é difícil avaliar a verdadeira qualidade e rigor dos procedimentos estatísticos aplicados. Dito de outra forma, por vezes é difícil aferir (porque 
não está explícito no trabalho revisto) se um determinado procedimento estatístico foi aplicado com rigor ou se os respetivos pressupostos de análise foram respeitados.

Finalmente, a presença de viés de publicação que, grosso modo, traduz a preferência dos autores de estudos empíricos e dos editores de revistas científicas pela publicação de resultados estatisticamente significativos ou tendentes à validação do conhecimento preexistente, constitui uma preocupação sempre presente (Card \& Krueger, 1995; Doucouliagos, Laroche \& Stanley, 2005; Humphreys, Reigel \& Epstein, 1995; Rosenthal, 1979; Stanley, 2008; Sterling, 1959, entre outros).

Algumas destas limitações podem ser atenuadas através da sua incorporação explícita no processo de revisão sistemática, mas outras não. Por exemplo, o investigador pode separar e analisar os estudos selecionados de acordo com os indicadores de qualidade que entenda serem pertinentes. Desde que deixe as suas opções bem explícitas, esta é uma opção valida, mas constituirá sempre uma das fragilidades da sua revisão sistemática. Já o viés de publicação é passível de ser detetado graficamente e testado estatisticamente (Stanley \& Doucouliagos, 2012), sobretudo quando a revisão sistemática se faz acompanhar de uma meta-análise.

\section{Etapas e procedimentos de uma revisão sistemática da literatura}

O paradigma sugerido por Cooper $(1982,2010)$ divide o processo de revisão sistemática da literatura em cinco a sete etapas, dependendo do que for incluído em cada uma delas. Aqui, optamos pela versão sugerida por Cooper e Hedges (2009), que adaptamos e apresentamos no Quadro 2. A leitura deste quadro revela que uma revisão sistemática da literatura deve conter:

- Uma questão de investigação explícita e claramente definida (etapa 1);

- Um procedimento de identificação e recolha de estudos, enquanto fontes primárias da revisão, que seja abrangente e sistemático (etapa 2);

- Critérios explícitos e estratégias replicáveis para efeitos de triagem e inclusão/exclusão de estudos (etapa 3);

- Quando justificável, critérios de avaliação e gradação da qualidade dos estudos incluídos (etapa 3);

- Estratégias explícitas e replicáveis de extração da informação de cada estudo relevante para a análise (etapa 3);

- Procedimentos adequados para efeitos de análise e apresentação dos dados e dos resultados (etapas 4 e 6);

- Uma interpretação dos resultados que decorra, de forma transparente e fluida, da análise dos dados (etapa 5);

- Um conjunto de implicações lógicas para a investigação futura e, quando pertinente, para a política económica e para as boas práticas das eventuais partes interessadas (etapa 5). 
Quadro 2. Etapas de uma revisão sistemática da literatura

\begin{tabular}{|c|c|c|}
\hline \multirow{2}{*}{ Etapa } & \multicolumn{2}{|c|}{ Conteúdo das etapas } \\
\hline & Questões analisadas & Observações \\
\hline $\begin{array}{c}\text { 1. Definição do } \\
\text { problema }\end{array}$ & $\begin{array}{l}\text { - Qual é a questão de investigação? } \\
\text { - Quais são as caraterísticas dos trabalhos } \\
\text { de investigação relevantes? }\end{array}$ & $\begin{array}{l}\text { - A questão de investigação deve ser tão } \\
\text { específica e focalizada quanto o possível; } \\
\text { - É necessário definir as variáveis e relações } \\
\text { de interesse, de modo a distinguir os } \\
\text { estudos relevantes dos irrelevantes. }\end{array}$ \\
\hline $\begin{array}{l}\text { 2. Recolha dos } \\
\text { dados }\end{array}$ & $\begin{array}{l}\text { - Que procedimentos vão ser utilizados } \\
\text { para encontrar os estudos relevantes? }\end{array}$ & $\begin{array}{l}\text { - Trata-se de identificar as fontes (e.g. } \\
\text { bases de dados, motores de pesquisa, } \\
\text { revistas, etc.) a pesquisar, os tipos de } \\
\text { evidência a recolher (e.g. artigos de } \\
\text { revistas, documentos de trabalho, atas de } \\
\text { conferências, etc.) e os termos ou } \\
\text { palavras-chave a utilizar na pesquisa. }\end{array}$ \\
\hline $\begin{array}{l}\text { 3. Avaliação dos } \\
\text { dados }\end{array}$ & $\begin{array}{l}\text { - Que estudos vão ser incluídos e excluídos } \\
\text { da síntese da literatura? } \\
\text { - Como é que a informação relevante, } \\
\text { recolhida a partir dos estudos incluídos, } \\
\text { vai ser codificada? }\end{array}$ & $\begin{array}{l}\text { - Trata-se de definir critérios para a inclusão } \\
\text { e exclusão dos trabalhos recolhidos; } \\
\text { - É necessário escolher qual é a informação } \\
\text { específica que vai ser recolhida a partir de } \\
\text { cada estudo (e.g. dimensão da amostra, } \\
\text { método estatístico, etc.). }\end{array}$ \\
\hline $\begin{array}{l}\text { 4. Apresentação e } \\
\text { análise dos } \\
\text { dados }\end{array}$ & $\begin{array}{l}\text { - Quais são os procedimentos ou métodos } \\
\text { estatísticos através dos quais os dados } \\
\text { recolhidos vão ser analisados? } \\
\text { - Como é que os dados recolhidos vão ser } \\
\text { apresentados e analisados do ponto de } \\
\text { vista gráfico e numérico? }\end{array}$ & $\begin{array}{l}\text { - Existem quatro abordagens básicas: } \\
\text { contagem de votos, combinação de } \\
\text { valores- } p \text {, determinação do tamanho do } \\
\text { efeito e análise de meta-regressão. }\end{array}$ \\
\hline $\begin{array}{l}\text { 5. Interpretação } \\
\text { dos resultados }\end{array}$ & $\begin{array}{l}\text { - Que conclusões podem ser retiradas da } \\
\text { análise de dados? } \\
\text { - Que procedimentos vão ser utilizados } \\
\text { para avaliar a sensibilidade e a robustez } \\
\text { das conclusões da meta-análise? } \\
\text { - Que implicações práticas resultam da } \\
\text { síntese da literatura? }\end{array}$ & $\begin{array}{l}\text { - Diz respeito à aplicabilidade dos } \\
\text { resultados, por força da evidência } \\
\text { encontrada. É necessário, por isso, } \\
\text { salientar o alcance e as limitações dos } \\
\text { resultados obtidos. }\end{array}$ \\
\hline $\begin{array}{l}\text { 6. Apresentação } \\
\text { dos resultados }\end{array}$ & $\begin{array}{l}\text { - A partir de toda a evidência recolhida, que } \\
\text { informação vai ser incluída no texto final? }\end{array}$ & $\begin{array}{l}\text { - A fidedignidade da síntese depende da } \\
\text { relevância e da qualidade da informação } \\
\text { apresentada. }\end{array}$ \\
\hline
\end{tabular}

Fonte: Adaptado de Cooper (1982; 2010) e Cooper e Hedges (2009).

Na primeira etapa, alguns dos aspetos sobre os quais é necessário refletir para efeitos de formulação da questão de investigação dizem respeito às variáveis dependentes e independentes a considerar, ao tipo de dados (corte transversal, séries temporais ou dados em painel), às metodologias de análise estatística e econométrica tidas como relevantes, entre outras.

A segunda etapa tem uma importância crítica, na medida em que uma das principais fontes de viés da revisão sistemática é o viés nos estudos incluídos. Por outras palavras, o enviesamento na amostra de estudos recolhidos constitui uma ameaça para todas as etapas subsequentes e para a própria validade da revisão sistemática. Algumas das estratégias a adotar tendo em vista reduzir a possibilidade de viés nesta etapa são as seguintes: pesquisar várias bases de dados, quer físicas, quer eletrónicas; incluir a "literatura cinzenta" (do inglês 
grey literature), o que corresponde a incluir documentos de trabalho, publicações em atas de conferências (entre outros trabalhos não publicados ou em fase de pré-publicação), para além de artigos publicados em revistas científicas; documentar rigorosamente o que foi pesquisado e como (nomeadamente palavras-chave escolhidas, datas de pesquisa abrangidas, etc.).

Igualmente importante é a terceira etapa, na qual se requer a definição de critérios para inclusão e exclusão de estudos. Dentro desses critérios encontram-se os tipos de estudos, tipos de publicações, variáveis analisadas, o horizonte temporal dos estudos publicados, etc. Também é possível triar os trabalhos com base em critérios qualitativos o que, contudo, levanta outras dificuldades (Valentine, 2009): critérios demasiado restritivos reduzem a quantidade de estudos incluídos e, por isso, restringem a capacidade de fazer generalizações; critérios demasiado inclusivos reduzem a fidedignidade dos resultados; a qualidade é, em si, fortemente subjetiva; podem existir discrepâncias entre o grau de qualidade de um trabalho como um todo e o grau de qualidade dos aspetos específicos que são relevantes para a revisão sistemática desejada.

Nesta terceira etapa, uma possibilidade é seguir as recomendações do Centre for Reviews and Dissemination (CRD, 2009). Estas recomendações dizem respeito às boas práticas para elaboração de revisões de literatura no âmbito das ciências da saúde, mas constituem um ponto de partida aplicável às ciências sociais. O CRD (2009) sugere que a triagem inicial dos trabalhos seja feita com base em cinco critérios que, adaptados ao nosso contexto, são os seguintes:

- População ou amostra analisada (corte transversal, dados em painel ou séries temporais);

- Variável dependente considerada;

- Variável independente principal ou de intervenção;

- Variáveis de controlo incluídas na análise;

- Metodologia de análise.

Os estudos selecionados que passam à etapa seguinte devem ter as caraterísticas desejadas em termos dos critérios que, destes cinco, sejam considerados relevantes.

Ainda nesta etapa é necessário extrair os dados relevantes para as etapas de análise dos mesmos. Esses dados devem ser codificados de forma sistemática e coerente e dizem respeito, entre outros aspetos, aos seguintes:

- Informação bibliográfica (autores, data de publicação, tipo de publicação, etc.);

- Caraterísticas do estudo (tipo de estudo, tipo de dados, unidades de medida das variáveis);

- Métodos de análise estatística ou de estimação econométrica (dimensão da amostra, metodologia, período analisado, etc.);

- Resultados apresentados (conclusão final, valores dos parâmetros estimados e respetivos desvios-padrão, resultados dos testes de causalidade, etc.). 
As etapas seguintes $(4,5$ e 6$)$ dizem respeito à análise dos dados propriamente dita. Nessas etapas, a colagem a orientações pré-definidas deve ser tanto mais rigorosa quanto mais a revisão sistemática venha a assentar em abordagens específicas de meta-análise. Isso significa que as revisões sistemáticas da literatura podem ser de dois tipos: com meta-análise e sem meta-análise (Booth, Papaioannou \& Sutton, 2012; Jesson, Matheson \& Lacey, 2011). Ou seja, podem ou não ser acompanhadas pela utilização de procedimentos estatísticos destinados a sintetizar os resultados empíricos dos vários estudos revistos (Koricheva \& Gurevitch, 2013). Por sua vez, a própria expressão “meta-análise” também pode ser interpretada de forma ambígua. Umas vezes designa a componente de síntese quantitativa de uma revisão sistemática da literatura; outras vezes designa a própria revisão sistemática, desde que ela utilize métodos quantitativos para sintetizar os resultados dos estudos revistos. Aqui, adotaremos a primeira aceção do termo.

Quando a revisão sistemática da literatura não é acompanhada por meta-análises, uma forma de concretizar as etapas 5 e 6 consiste em avaliar em que medida é que as diferenças existentes entre as conclusões dos estudos revistos podem ser explicadas pelas caraterísticas da informação codificada e organizada nas etapas precedentes, nomeadamente a 3 e a 4 . Essa avaliação pode ser complementada pelas análises da validade, fiabilidade e aplicabilidade dos estudos revistos, tal como sugerido por Ugur e Dasgupta (2011, citado em Ugur, 2014: 486, nota 1). As ditas análises consistem em colocar, relativamente a cada estudo, as seguintes questões:

- Validade do construto: a relação entre as variáveis dependente e independentes é teorizada e modelizada de forma coerente com a literatura disponível?

- Validade do método: a metodologia de análise empírica é consistente com as meIhores práticas disponíveis?

- Fiabilidade dos dados: a fiabilidade e as limitações dos dados são discutidas?

- Fiabilidade da estimação: são incluídas variáveis de controlo relevantes?

- Fiabilidade dos resultados: são feitas análises de sensibilidade e de robustez aos resultados?

- Aplicabilidade das conclusões: as conclusões obtidas são aplicáveis a conjuntos ou amostras alargadas de países, incluindo a países não considerados nas amostras efetivamente analisadas?

Trata-se, por isso, de avaliar as várias vertentes da validade, fiabilidade e aplicabilidade dos estudos analisados, tendo em vista assegurar as maiores objetividade e replicabilidade possíveis no ato de interpretação e análise dos dados. Naturalmente, a qualidade das respostas dadas nesta etapa depende não só da qualidade e do grau de concretização das etapas anteriores, mas também da verticalidade e espírito crítico do investigador. Nesse sentido, ele deve demonstrar claramente como é que a resposta a cada questão decorre naturalmente das análises efetuadas nas etapas anteriores. 


\section{Conclusões}

As revisões sistemáticas da literatura constituem uma estratégia de síntese do conhecimento já há muito conhecida e utilizada no âmbito das ciências da saúde e da educação. Só muito recentemente começaram a ser alargadas ao âmbito das ciências sociais. O caráter padronizado das revisões sistemáticas confere-lhes uma maior objetividade e permite a respetiva replicabilidade o que, por sua vez, assegura a fidedignidade das conclusões obtidas. Os procedimentos apresentados ao longo deste trabalho constituem um modelo que pode ser aplicado pelos investigadores dos mais diversos ramos das ciências sociais sempre que esteja em causa a necessidade de apresentar balanços do conhecimento disponível, complementados ou não por estudos de meta-análise.

Neste trabalho procurámos apresentar um texto suficientemente conciso, mas também suficientemente claro e objetivo, tendo em vista motivar a ação. Paralelamente, ao indicar as principais referências relativas à aplicação da metodologia de revisão sistemática da literatura, acabámos dar ao leitor as ferramentas necessárias ao aprofundamento de cada um dos aspetos abordados aqui.

A investigação futura pode e deve seguir em pelo menos duas direções interligadas. Por um lado, sempre que se justifique, é necessário fazer uma transposição e adaptação dos procedimentos de revisão sistemática da literatura para cada uma das áreas das ciências sociais, tendo em vista dar resposta às especificidades que possam ser relevantes. Contudo, é imperioso que não se percam as traves mestras da metodologia, que são as que apresentámos aqui. Por outro lado, é desejável que os trabalhos de revisão de literatura, em quaisquer áreas das ciências sociais, venham a fazer uso, cada vez mais, deste tipo de abordagem. Isto porque a sua natureza replicável permite que os públicos-alvo interessados possam assumir uma posição crítica mais bem fundamentada a respeito dos balanços do conhecimento que lhes sejam apresentados. Se assim for, torna-se mais fácil discernir e discutir quer os pontos de concordância, quer os de discordância, bem como detetar os rumos mais viáveis a dar à investigação futura. Por outras palavras, torna-se mais fácil fazer avançar o conhecimento em cada uma das áreas de interesse.

\section{Referências}

Barnes, D. \& Bero, L. (1998). Why review articles on the health effects of passive smoking reach different conclusions. Journal of the American Medical Association, 279(19), 1566-1570. DOI: 10.1001/jama.279.19.1566.

Booth, A., Papaioannou, D. \& Sutton, A. (2012). Systematic approaches to a successful literature review. Londres: Sage.

Card, D. \& Krueger, A. (1995). Time-series minimum-wage studies: A meta-analysis. American Economic Review, 85(2), 238-243. DOI: 10.2307/2117925.

Centre for Reviews and Dissemination [CRD] (2009). Systematic reviews: CRD's guidance for undertaking reviews in health care. Heslington, York, UK: University of York.

Cooper, H. (1982). Scientific guidelines for conducting integrative research review. Review of Educational Research, 52(2), 291-302. DOI: 10.3102/00346543052002291.

Cooper, H. (2010). Research synthesis and meta-analysis: A step-by-step approach. Thousand Oaks, CA: Sage. 
Cooper, H. \& Hedges, L. (2009). Research synthesis as a scientific process. In H. Cooper, L. Hedges \& J. Valentine (Eds.) The Handbook of Research Synthesis and Meta-analysis (pp. 3-18). Nova Iorque: Russell Sage Foundation.

Crossan, M. \& Apaydin, M. (2010). A multi-dimensional framework of organizational innovation: A systematic review of the literature. Journal of Management Studies, 47(6), 1154-1191, DOI: 10.1111/j.1467-6486.2009.00880.x.

Doucouliagos, H., Laroche, P. \& Stanley, T. (2005). Publication bias in union-productivity research? Relations Industrielles, 60(2), 320-347+372. DOI: 10.2307/23078055.

Gomezelj, D. (2016). A systematic review of research on innovation in hospitality and tourism. International Journal of Contemporary Hospitality Management, 28(3), 516-558, DOI: 10.1108/ijchm-10-2014-0510

Higgins, J. \& Green, S. (2008) Cochrane handbook for systematic reviews of interventions. CA: John Wiley \& Sons Inc.

Holland, N. (1948). Extra-oral pin control of fragments of fractured mandibles; a systematic review of cases. British Dental Journal, 84(3), 47-52.

Humphreys, R., Reigel, D. \& Epstein, F. (1995). The editors' labours: Separating the wheat from the chaff. Pediatric Neurosurgery, 22(5), 223-227. DOI: 10.1159/000120905.

Hunter , J., Schmidt, F. \& Hunter, R. (1979). Differential validity of employment tests by race: A comprehensive review and analysis. Psychological Bulletin, 86(4), 721-735. DOI: 10.1037/00332909.86.4.721.

Jesson, J., Matheson, L. \& Lacey, F. (2011). Doing your literature review: Traditional and systematic techniques. Londres: Sage.

Koricheva, J. \& Gurevitch, J. (2013). Place of meta-analysis among other methods of research synthesis. In J. Koricheva, J. Gurevitch \& K. Mengersen (Eds.) Handbook of Meta-analysis in Ecology and Evolution. Princeton: Princeton University Press. DOI: 10.23943/princeton/9780691137285.003.0001

Lim, K. \& Brooks, R. (2011). The evolution of stock market efficiency over time: A survey of the empirical literature. Journal of Economic Surveys, 25(1), 69-108. DOI: 10.1111/j.1467-6419.2009.00611.x.

Moher, D., Liberati, A., Tetzlaff, J., Altman, D.G., Altman, D., Antes, G., ...\& \& Magrini, N. (2009). Preferred reporting items for systematic reviews and meta-analyses: The PRISMA statement. Annals of Internal Medicine, 151(4), 264-269. DOI: 10.1371/journal.pmed.1000097.

Peloza, J. \& Shang, J. (2011). How can corporate social responsibility activities create value for stakeholders? A systematic review. Journal of the Academy of Marketing Science, 39(1), 117-135. DOI: 10.1007/s11747-010-0213-6.

Perkmann, M., Tartari, V., McKelvey, M., Autio, E, Broström, A., D'Este, P., ... Sobrero, M. (2013). Academic engagement and commercialisation: A review of the literature on universityindustry relations. Research Policy, 42(2), 423-442. DOI: 10.1016/j.respol.2012.09.007.

Pittaway, L. \& Cope, J. (2007). Entrepreneurship education: A systematic review of the evidence. International Small Business Journal, 25(5), 479-510, DOI: 10.1177/0266242607080656.

Pittaway, L., Robertson, M., Munir, K., Denyer, D. \& Neely, A. (2004). Networking and innovation: A systematic review of the evidence. International Journal of Management Reviews, 5-6(3-4), 137168. DOI: $10.1111 /$ j.1460-8545.2004.00101.x.

Rosenthal, R. (1979). The file drawer problem and tolerance for null results. Psychological Bulletin, 86(3), 638-641. DOI: 10.1037/0033-2909.86.3.638.

Shadish, W. (2015). Introduction to the special issue on the origins of modern meta-analysis. Research Synthesis Methods, 6(3), 219-220. DOI: 10.1002/jrsm.1148.

Stanley, T. (2008). Meta-regression methods for detecting and estimating empirical effects in the presence of publication selection. Oxford Bulletin of Economics and Statistics, 70(1), 103-127. DOI: $10.1111 / \mathrm{j} .1468-0084.2007 .00487 . x$.

Stanley, T. \& Doucouliagos, H. (2012) Meta-regression analysis in economics and business. Oxfordshire: Routledge. 
Sterling, T. (1959). Publication decisions and their possible effects on inferences drawn from tests of significance - Or vice versa. Journal of the American Statistical Association, 54(285), 30-34. DOI: 10.1080/01621459.1959.10501497.

Suri, H. (2000). A critique of contemporary methods of research synthesis. Post-Script, 1(1), 49-55.

Ugur, M. (2014). Corruption's direct effects on per-capita income growth: A meta-analysis. Journal of Economic Surveys, 28(3), 472-490. DOI: 10.1111/joes.12035.

Valentine, J. (2009). Judging the quality of primary research. In H. Cooper, L. Hedges \& J. Valentine (Eds.) The Handbook of Research Synthesis and Meta-Analysis (pp. 129-166). Nova Iorque: Russel Sage Foundation.

van Rooyen, C., Stewart, R. \& de Wet, T. (2012). The Impact of Microfinance in Sub-Saharan Africa: A Systematic Review of the Evidence. World Development, 40(11) 2249-2262, DOI: 10.1016/j.worlddev.2012.03.012.

Weed, M. (2006). Sports tourism research 2000-2004: A systematic review of knowledge and a metaevaluation of methods. Journal of Sport and Tourism, 11(1), 5-30. DOI: $10.1080 / 14775080600985150$.

Zhang, X., Song, H. \& Huang, G. (2009). Tourism supply chain management: A new research agenda. Tourism Management, 30(3), 345-358, DOI: 10.1016/j.tourman.2008.12.010.

NINO FONSECA é doutorado em Economia pela Universidade da Extremadura. É professor adjunto no Instituto Politécnico de Viana do Castelo, onde leciona Economia do Turismo e Macroeconomia aos cursos de licenciatura em Turismo e em Gestão. Os seus interesses de investigação incluem a análise de meta-regressão, a causalidade em Economia, o crescimento económico e a economia do turismo. ORCID: 0000-0003-3177-0852. Endereço institucional: Instituto Politécnico de Viana do Castelo, Escola Superior de Tecnologia e Gestão, Avenida do Atlântico, n. ${ }^{\circ} 644$, 4900-348 Viana do Castelo, Portugal.

MARCELINO SÁNCHEZ-RIVERO, PhD, é professor titular de métodos quantitativos na Universidade da Extremadura. Os seus interesses de investigação incluem a análise estatística de tabelas de contingência, os modelos de estrutura latente, a estatística e econometria espaciais, a análise do comportamento do turista e a análise da sustentabilidade e competitividade do turismo. ORCID: 00000003-3988-6278. Endereço institucional: Faculdade de Ciências Económicas e Empresariais, Departamento de Economia, Universidade da Extremadura, Avenida de Elvas, s/n, 06006, Badajoz, Espanha.

Submetido em 17 julho 2019

Aceite em 28 outubro 2019 